TRANSACTIONS OF THE

AMERICAN MATHEMATICAL SOCIETY

Volume 351, Number 6, Pages $2233-2255$

S 0002-9947(99)02343-0

Article electronically published on February 4, 1999

\title{
DERIVATIVES OF WRONSKIANS WITH APPLICATIONS TO FAMILIES OF SPECIAL WEIERSTRASS POINTS
}

\author{
LETTERIO GATTO AND FABRIZIO PONZA
}

\begin{abstract}
Let $\pi: \mathfrak{X} \longrightarrow S$ be a flat proper family of smooth connected projective curves parametrized by some smooth scheme of finite type over $\mathbb{C}$. On every such a family, suitable derivatives "along the fibers" (in the sense of Lax) of the relative wronskian, as defined by Laksov and Thorup, are constructed. They are sections of suitable jets extensions of the $g(g+1) / 2$-th tensor power of the relative canonical bundle of the family itself.

The geometrical meaning of such sections is discussed: the zero schemes of the $(k-1)$-th derivative $(k \geq 1)$ of a relative wronskian correspond to families of Weierstrass Points (WP's) having weight at least $k$.

The locus in $M_{g}$, the coarse moduli space of smooth projective curves of genus $g$, of curves possessing a WP of weight at least $k$, is denoted by $w t(k)$. The fact that $w t(2)$ has the expected dimension for all $g \geq 2$ was implicitly known in the literature. The main result of this paper hence consists in showing that $w t(3)$ has the expected dimension for all $g \geq 4$. As an application we compute the codimension 2 Chow $(Q$-)class of $w t(3)$ for all $g \geq 4$, the main ingredient being the definition of the $k$-th derivative of a relative wronskian, which is the crucial tool which the paper is built on. In the concluding remarks we show how this result may be used to get relations among some codimension 2 Chow $\left(Q\right.$-) classes in $M_{4}(g \geq 4)$, corresponding to varieties of curves having a point $P$ with a suitable prescribed Weierstrass Gap Sequence, relating to previous work of Lax.
\end{abstract}

\section{INTRODUCTION}

0.1. The main goal that motivated the investigations which led to this paper was to deal with loci of the (coarse) moduli space $M_{g}$ of the smooth projective curves of genus $g$ (defined over an algebraically closed field of characteristic zero), whose points correspond to curves possessing some special Weierstrass points.

We are, in fact, especially interested in the computation of the classes in the Chow ring of $M_{g}$ with rational coefficients (as defined by Mumford in [Mu2], see section 1.5) of some of such loci. To achieve this goal we have been in some sense forced to introduce and to study a locus in $M_{g}$ which does not seem to have been considered in the earlier literature. In the moduli space $M_{g}$ define:

$$
w t(k)=\left\{[C] \in M_{g} / C \text { has a point } P \text { such that } w t(P) \geq k\right\},
$$

Received by the editors February 5, 1997.

1991 Mathematics Subject Classification. Primary 14H10, 14H15, 14H55, 14H99.

Key words and phrases. Relative wronskians, derivatives of relative wronskians, families of Weierstrass points, moduli spaces of curves, Chow classes in $M_{g}$.

Work partially supported by GNSAGA-CNR, MURST and by Dottorato di Ricerca in Matematica, Consorzio Universitario Torino-Genova. 
i.e. the locus of (isomorphism classes of) curves in $M_{g}$ possessing a Weierstrass point with weight at least $k$. In order to study such a locus, above defined only as a set, one needs to put on it a reasonable structure of scheme and to study how its dimension varies, by varying $k$. For instance it is quite clear that $w t(1)$ must be all of $M_{g}$, a fancy way of saying that every curve of genus $g \geq 2$ has at least 1 Weierstrass point.

0.2. In order to put on $w t(k)$ a scheme structure, one has to deal with some natural but very useful tools, developed in section 2, whose construction, indeed, forms the conceptual core of the paper. As is well known, the Weierstrass Points (WP's in the following) on a smooth curve of genus $g$, can be detected as the zero-locus of the wronskian section of the bundle $K^{\otimes \frac{g(g+1)}{2}}, K$ being the canonical sheaf of the curve. Such a picture can be extended in a functorial way to families of curves. Namely, if $\pi: \mathfrak{X} \longrightarrow S$ is a smooth curve over $S$ ( $S$ taken to be, in this paper, a smooth scheme of finite type over $\operatorname{Spec}(\mathbb{C}))$, then a wronskian section associated to the family can be defined. It is a section of the bundle $K_{\pi}{ }^{\otimes \frac{g(g+1)}{2}} \otimes\left(\pi^{*} \bigwedge^{g} \pi_{*} K_{\pi}\right)^{\vee}$, where $K_{\pi}$ is the relative canonical sheaf of the family $\pi$ (see section 2 for details). In [LT1] and [LT2] such a relative wronskian is defined regardless of the characteristic of the ground field the curves are defined over. What we do, in section 2 , is to consider not only the relative wronskian but also its derivatives in the following sense. If $\mathbb{W}_{\pi}$ is the wronskian associated to the family $\pi$, then its $k$-th derivative is a section $D^{k} \mathbb{W}_{\pi}$ of the bundle $J_{\pi}^{k}\left(K_{\pi} \otimes^{\frac{g(g+1)}{2}}\right) \otimes\left(\pi^{*} \bigwedge^{g} \pi_{*} K_{\pi}\right)^{\vee}$ and has the property that, restricted to a fiber $\mathfrak{X}_{s}$ of the family, it is roughly described, in a local coordinate of the fiber, by the $k$-th derivative of the wronskian with respect to the local coordinate itself. Here $J_{\pi}^{k} \mathcal{L}(\mathcal{L}$ a line bundle over $\mathfrak{X})$ means the $k$-th jets extension of $\mathcal{L}$ along the fibers of $\pi$. This has been already used by Lax in [L1] using Patt local coordinates [Pa]. The sections $D^{k} \mathbb{W}_{\pi}$ allow us to define other loci in the moduli space, with a precise description of their scheme structure. These are precisely the loci $w t(k)$, for $k \geq 1$, that we have mentioned at the beginning of this introduction. As a matter of fact, the scheme structure of such loci is morally described by the zero scheme of the section $D^{k-1} \mathbb{W}$ on $M_{g, 1}$.

0.3. Once we have defined the locus $w t(k)$, for a given $k$, as the image via a proper morphism of the zero-scheme of a section of a vector bundle, its class in the intersection ring of $M_{g}$, whatever that means, should correspond to the top Chern class of the bundle itself. However, in order to give a geometrical meaning to such an intersection class, one must primarily check that $w t(k)$ has the expected codimension $k-1$ in $M_{g}$. In general this turns out to be false. It is very easy to show, for instance, that $w t(4)$ in $M_{5}$ (cf. the counterexample 3.4) has not the expected codimension. The existence of such counterexamples motivates the search for the values of $g$ for which $w t(k)$ has the expected dimension in $M_{g}$. In this paper we shall prove that:

Theorem 3.6. The locus wt(2) is empty in $M_{2}$. For $g>2$, each irreducible component of $w t(2)$ has the expected dimension $3 g-4$.

This theorem might be considered well known, because $w t(2)$ is strongly related to two other natural divisors, defined in terms of curves possessing some special Weierstrass points, which have been extensively studied in the literature. The first one is the locus $\mathbb{D}_{g-1}$ of the curves having a Weierstrass point whose first non-gap 
is $g-1$. The second one is the locus $E(1)$ of the curves possessing a Weierstrass point of type $g+1$ : a point $P$ of a curve $C$ of genus $g$ is said to be of type $g+k$ $(k \geq 1)$ if there exists a non-zero canonical divisor containing $n P$, with $n \geq g+k$. As a matter of fact, it was already clear that $w t(2)$, as a set, is the union of $E(1)$ and $\mathbb{D}_{g-1}(\mathrm{cf}$. $[\mathrm{Cu}]$, p. 339). Thanks to the new notion of the derivative of the relative wronskian, and the consequent definition of a scheme structure on $w t(2)$, we can make sharper this result. In fact we may prove, by direct computation, that:

4.6. Theorem. For $g \geq 4$, the following equality holds in the group $\operatorname{Pic}\left(M_{g}\right) \otimes \mathbb{Q}$ :

$$
[w t(2)]=[E(1)]+\left[\mathbb{D}_{g-1}\right]
$$

while, for $g=3$, one has:

$$
[w t(2)]=[W(1,2,5)]+16\left[H_{3}\right]
$$

in $\operatorname{Pic}\left(M_{3}\right) \otimes \mathbb{Q}$, where

$$
W(1,2,5)=\left\{[C] \in M_{3} / C \text { has a Weierstrass point with }\langle 1,2,5\rangle \text { as WGS }\right\} .
$$

Of course, the previous result says nothing about the scheme theoretical relation between $w t(2), E(1)$ and $\mathbb{D}_{g-1}$. However, one may prove (see Remark 4.7) that things are as nice as one may hope: in [Ga3] it is shown that, for $g \geq 4$, wt(2) is actually the scheme theoretic union of $E(1)$ and $\mathbb{D}_{g-1}$, a fact which turns out to be very useful for performing computations in $\operatorname{Pic}\left(\bar{M}_{g}\right) \otimes \mathbb{Q}$, the rational Picard group of the Deligne-Mumford compactification of $M_{g}$ ([DM]).

0.4. Beside the above quoted results, it should be quite clear that things become more and more interesting when working in higher codimension. However, even if it is very easy to compute the top Chern class of the bundle $J_{\pi}^{k-1}\left(K_{\pi} \otimes \frac{g(g+1)}{2}\right) \otimes$ $\left(\pi^{*} \bigwedge^{g} \pi_{*} K_{\pi}\right)^{\vee}$, as already remarked, such a class corresponds with the actual class of the subscheme $[w t(k)]$ only if $[w t(k)]$ has the expected codimension in $M_{g}$. A natural problem then arises: given $k \geq 3$ find the least positive integer $g(k)$, such that for each $g \geq g(k), w t(k)$ has the expected codimension in $M_{g}$. The Referee remarks that one should expect, according to results of Eisenbud and Harris ([EH]), that $w t(k)$ will have the expected dimension if $g \geq 2 k$. Our first contribution to such a natural problem is provided by what may be considered the main result of the paper.

Theorem 3.7. Each irreducible component of wt(3) has the expected dimension $3 g-5$, for each $g \geq 4$.

The above statement immediately implies that the $Q$-class of $w t(3)$ in $A^{*}\left(M_{g}\right)$ $(g \geq 4)$ can be computed by computing the push down of the localized top Chern class ([Fu], p. 244) of the section $D^{2} \mathbb{W}_{\pi}$ (see section 4 for details). Simple calculations yield:

Proposition 4.9. The $Q$-class of $w t(3)$ in $A^{*}\left(M_{g}\right)$ is given by:

$$
\begin{aligned}
{[w t(3)] } & =\frac{1}{8} g(g+1)\left(g^{2}+g+2\right)\left(g^{2}+g+4\right) \kappa_{2}+ \\
& -\frac{1}{4}\left[2 g(g+1)\left(g^{2}+g+3\right)+\left(g^{2}+g+2\right)\left(g^{2}+g+4\right)\right] \kappa_{1} \lambda .
\end{aligned}
$$


Such a computation gives us some qualitative informations on curves of genus 4 . In fact, if we denote by $E(2)$ the locus of (isomorphism classes of) curves of $M_{g}$ possessing a Weierstrass point of type $g+2$ (cf. 0.3 of this introduction), it turns out that, if $g=4, w t(3)$ and $E(2)$ are equal as sets. However, their scheme structure is certainly different, as one may immediately see by comparing the $Q$-class of $E(2)$ (known in the literature) with the $Q$-class of $w t(3)$, computed in our paper. We also indicated how it should be possible to find the classes of the loci $[W(1,2,3,7)]_{Q}$ and $[W(1,2,4,7)]_{Q}$ in $M_{4}$. This question seems to us quite interesting for the reason explained below (see also the concluding remarks, at the end of the paper).

0.5. As is well known, one of the ways to stratify $M_{g}$ is to look at those subvarieties of it whose (isomorphism classes of) curves have special Weierstrass points. From this point of view there are important results by Arbarello [A] and Diaz [D2]. For low genera, moreover, it is quite possible to study the geometry of the loci in $M_{g}$ characterized by such properties. For instance, in $M_{3}$ we can distinguish two divisors, the one corresponding to the hyperelliptic curves and the one corresponding to curves having a WP with $\langle 1,2,5\rangle$ as a Weierstrass gap sequence. The classes in the Picard group of $M_{3}$ (with $\mathbb{Q}$-coefficients) of both loci are well known and computed in several places. But, in general, it is not at all clear when, given a sequence of $g$ integers, such that its complement in $\mathbb{N}$ is a semigroup $\Gamma$ (see section 1 for basic definitions), there exists a smooth genus $g$ curve with a point $P$ having $\Gamma_{P}=\Gamma$. A difficult problem is also to study the dimension, when non empty, of the closure of the loci $W\left(1, n_{2}, \ldots, n_{g}\right)$ defined in (0.1). But, as already said, for low genera this can be done in a relatively easy way. If $g=4$, for instance, all the possible sequences of integers $\left\langle 1, n_{2}, n_{3}, n_{4}\right\rangle$, such that $\Gamma=\mathbb{N} \backslash\left\{1, n_{2}, n_{3}, n_{4}\right\}$ is an additive sub-semigroup of $\mathbb{N}$, actually occur as WGSs of some point $P$ belonging to some smooth projective curve of genus 4 . Moreover in [L2], R.F. Lax shows that the closures in $M_{4}$ of all the corresponding subvarieties $W\left(1, n_{2}, n_{3}, n_{4}\right)$ have the expected codimension inside $M_{4}$. By previous work of Diaz [D1], the classes of $W(1,2,3,6)$ and of $W(1,2,4,5)$ were computed in $\operatorname{Pic}\left(M_{4}\right) \otimes \mathbb{Q}$. Moreover the $(Q$-)class of $W(1,3,5,7)$ (the hyperelliptic locus) was computed by Mumford in [Mu2], and our original task was to compute the $Q$-classes of the two remaining loci, $W(1,2,3,7)$ and $W(1,2,4,7)$, to refine in a sense, the picture drawn by Lax in $[\mathrm{L} 2]$.

0.6. The strategy we suggest for achieving such a goal is as follows. Since $w t(3)$ has the expected codimension inside $M_{4}$, one first computes the class of $w t(3)$ in $A^{2}\left(M_{4}\right)$ and observes that it is a linear combination (with integral coefficients) of the class of the hyperelliptic locus and of the classes of $W(1,2,3,7)$ and $W(1,2,4,7)$ respectively. Secondly we use results by Diaz: the class $[E(2)]$ defined in [D2] (cf. section 1.4) can be computed by Porteous' formula ([ACGH]) and it is an integral linear combination of the class of the hyperelliptic locus, of $[W(1,2,3,7)]$ and of $[W(1,2,4,7)]$, too. We get in this way a system of two linear equations in the two unknowns $[W(1,2,3,7)]$ and $[W(1,2,4,7)]$. Once one knows the coefficients which $\left[H_{4}\right],[W(1,2,4,7)]$ and $[W(1,2,3,7)]$ appear with, one may solve the system to determine the two unknown classes. Even if we do not know exactly such coefficients, we are however able to give lower bounds on them, thanks once more to the notion of the $k$-th derivative of a relative wronskian. 
0.8. Actually, we think it is worth saying that we came to study the derivatives of wronskians within the more general framework of what we called, in an unpublished preprint $([\mathrm{GP}])$, the generalized wronskian sections. They are distinguished sections of some vector bundles similarly called Generalized Wronskian Bundles. The full theory of such bundles is developed in [Po1] and we already gave a brief and very informal account of it in [GP]. Another paper on the subject ([Po2]) is in preparation. This paper, together with [Ga3], is one of the first applications of such a theory and our hope is that the use of more general wronskian sections, not recoverable as derivatives of wronskians in the sense of section 2, may be useful to get new results about relations between the tautological classes, according to a program drawn by C. Faber few years ago and explained in [Fa2].

0.9. The paper is organized as follows. Section 1 is devoted to set up the basic notation and preliminaries. For the notation not included in such a section we refer, as usual, to the Hartshorne book [Ha]. As already said, section 2 is the core of the paper and the most important for the applications: there we consider the derivatives of the relative wronskian seen, like in [LT1] for the usual wronskians, as suitable maps of vector bundles. The new results (Theorems 3.7 and 4.6, Proposition 4.9) are proven in sections 3 and 4. The first part of section 4 has also a "pedagogical" purpose: some examples already worked out in the literature are revisited, to "emotionally" convince the reader that our tools work properly.

Acknowledgment. We are warmly grateful to S. Greco, G. P. Pirola and the unknown referee for useful remarks and to C. Faber for having pointed out a wrong reference in the quoted preprint [GP]. Special thanks are due to Marc Coppens, whose e-mail comments improved our understanding of the subject.

\section{Preliminaries and notation}

1.1. In this paper we shall deal with smooth projective algebraic curves of genus $g \geq 2$ over the complex field $\mathbb{C}$ (Compact Riemann Surfaces) or with suitable families of them. A point $P \in C$ is said to be a Weierstrass point (WP in the following) if and only if:

$$
h^{0}(C, K(-g P)) \geq 1 .
$$

Let $n \geq 1$ be a positive integer such that:

$$
h^{0}(C, K(-(n-1) P))>h^{0}(C, K(-n P)) .
$$

Then $n$ is said to be a Weierstrass gap at the point $P$. It is easy to show that each point of $C$ admits exactly $g$ Weierstrass gaps, $1=n_{1}<\ldots<n_{g} \leq 2 g-1$. The increasing sequence of integers $\left\langle 1=n_{1}, \ldots, n_{g} \leq 2 g-1\right\rangle$ is hence said to be the Weierstrass Gap Sequence (WGS in the following) at $P$. An integer $n \in \mathbb{N}$ is said to be a non-gap at $P$ if and only if it is not a gap at $P$. It is easy to see, by using the Riemann-Roch formula, that $n \in \mathbb{N}$ is a non-gap iff there exists a meromorphic function $f$ on $C$ which is holomorphic on $C \backslash\{P\}$ having at $P$ a pole of order exactly $n([\mathrm{Gu}],[\mathrm{Fo}])$. Using these facts one can prove that the complement in $\mathbb{N}$ of the WGS is an additive subsemigroup of $\mathbb{N}, \Gamma_{P}$, called the Weierstrass semigroup at $P$. A point $P \in C$ is a Weierstrass point iff its WGS is not $\langle 1,2, \ldots, g\rangle$. The 
weight of the WGS at $P$ or, equivalently, the weight of the semigroup $\Gamma_{P}$ is defined to be:

$$
w t\left(\Gamma_{P}\right)=w t(W G S)=\sum_{i=1}^{g}\left(n_{i}-i\right)
$$

1.2. The Weierstrass weight of a point $P \in C, w t(P)$, is defined to be the order of vanishing of the wronskian section $\mathbb{W} \in H^{0}\left(C, K^{\otimes \frac{g(g+1)}{2}}\right)$ at $P$. Let $\boldsymbol{\omega}=$ $\left(\omega_{1}, \ldots, \omega_{g}\right)$ be a basis of $H^{0}(C, K)$, and let $(U, z)$ be a local coordinate chart trivializing $K$ and such that $z(P)=0$. This means that, locally, $\omega_{i}=u_{i}(z) d z$. Then the wronskian is locally defined as (see, e.g., $[\mathrm{Fo}],[\mathrm{Gu}]$ ):

$$
\mathbb{W}_{\left.\right|_{U}}(z)=\left|\begin{array}{ccc}
u_{1}(z) & \ldots & u_{g}(z) \\
u_{1}^{\prime}(z) & \ldots & u_{g}^{\prime}(z) \\
\vdots & \ddots & \vdots \\
u_{1}^{(g-1)}(z) & \ldots & u_{g}^{(g-1)}(z)
\end{array}\right|
$$

where the derivatives of the local functions $u_{i}$ 's are taken with respect to the local coordinates. For short, we shall often write the right hand side of (1.4) as

$$
\mathbf{u}(z) \wedge \mathbf{u}^{\prime}(z) \wedge \ldots \wedge \mathbf{u}^{(g-1)}(z)
$$

which is nothing but an abbreviated notation for:

$$
\operatorname{det}\left(\begin{array}{c}
\mathbf{u}(z) \\
\vdots \\
\mathbf{u}^{(g-1)}(z)
\end{array}\right),
$$

having set $\mathbf{u}=\left(u_{1}, \ldots, u_{g}\right) \in O_{C}(U)^{\oplus g}$. The order of vanishing at $P$ of the wronskian section $\mathbb{W}$ is the order of vanishing of $\mathbb{W}_{\left.\right|_{U}}(z)$ at $z=0$. One can show that such a definition works well in the sense that such a vanishing order depends neither on the particular basis chosen for the holomorphic differentials nor on the choice of local coordinate around $P$. A classical result states that $w t(P)=w t\left(\Gamma_{P}\right)$ (see $[\mathrm{Gu}],[\mathrm{ACGH}])$.

1.3. The Brill-Nöther matrix (or, rather, its transpose: see [ACGH]) of the divisor $d P(d \in \mathbb{N}, P \in C)$ is the $d \times g$ matrix with complex entries defined as:

$$
\mathcal{B N}_{U}(d P)=\left(\begin{array}{c}
\mathbf{u}(0) \\
\mathbf{u}^{\prime}(0) \\
\vdots \\
\mathbf{u}^{(d-1)}(0)
\end{array}\right)
$$

where the subscript $U$ means that the matrix has been computed with respect to the local chart $(U, z)$ and $\mathbf{u}^{(h)}(0)$ stands for the $h$-th derivative of the local holomorphic $\mathbb{C}^{g}$-valued function $\mathbf{u}$, with respect to the parameter $z$, evaluated at $z=0$. Though $\mathcal{B N}_{U}(d P)$ changes by changing the local chart $(U, z)$ and the basis $\boldsymbol{\omega}$ of $H^{0}(C, K)$, it is a standard (and easy to check) fact that its rank does not (see [ACGH] or $[\mathrm{Gu}]$ ). Hence the rank of the Brill-Nöther matrix is a datum attached to the point only. For such a reason, we shall write the Brill-Nöther matrix in a more intrinsic 
way, by skipping the dependence from $U$, being interested solely in its rank:

$$
\mathcal{B N}(d P)=\left(\begin{array}{c}
\boldsymbol{\omega}(P) \\
D \boldsymbol{\omega}(P) \\
\vdots \\
D^{d-1} \boldsymbol{\omega}(P)
\end{array}\right):=\left(\begin{array}{ccc}
\boldsymbol{\omega}_{1}(P) & \ldots & \boldsymbol{\omega}_{g}(P) \\
\left(D \boldsymbol{\omega}_{1}\right)(P) & \ldots & \left(D \boldsymbol{\omega}_{g}\right)(P) \\
\vdots & \ddots & \vdots \\
\left(D^{d-1} \boldsymbol{\omega}_{1}\right)(P) & \ldots & \left(D^{d-1} \boldsymbol{\omega}_{g}\right)(P)
\end{array}\right)
$$

so that $\rho(d P):=r k(\mathcal{B N}(d P))=r$ if and only if $r k\left(\mathcal{B N}_{U}(d P)\right)=r$ for one (and hence for all) local chart $(U, z)$ around $P \in C$. A well known fact $([\mathrm{Gu}])$ says that:

$$
h^{0}\left(C, O_{C}(d P)\right)=d+1-\rho(d P) .
$$

Using this fact and the Riemann-Roch formula, one concludes that:

$$
\rho(2 g P)=g \text {. }
$$

1.4. We fix another piece of notation following [D2]. In $M_{g, 1}$, the coarse moduli space of pointed curves of genus $g$ (often called the "universal curve" over $M_{g}$ ), we define the locus:

$$
V\left(1, n_{2}, \ldots, n_{g}\right)=\left\{(C, P) \in M_{g, 1} / W G S(P)=\left\langle 1, n_{2}, \ldots, n_{g}\right\rangle\right\} .
$$

We also define some loci in $M_{g}$, the coarse moduli space of smooth genus curves of genus $g$. We set:

$W\left(1, n_{2}, \ldots, n_{g}\right)=\left\{[C] \in M_{g} / C\right.$ has a point $P$ with $\left.W G S(P)=\left\langle n_{1}, n_{2}, \ldots, n_{g}\right\rangle\right\}$,

Moreover we say that a point $P$ is a WP of type $k$ if and only if:

$$
h^{0}(C, k P) \geq \max (2, k-g+2) .
$$

This means, if $2 \leq k \leq g$, that $P$ is of type $k$ if and only if $h^{0}(C, k P) \geq 2$, while a point $P$ is of type $g+k$ if and only if $h^{0}(C,(g+k) P) \geq k+2$. We define the loci $\mathbb{D}_{k}, E(k)$ and $F(k)$ as follows (cf. [D2]). For $2 \leq k \leq g$

$$
\mathbb{D}_{k}=\left\{[C] \in M_{g} / C \text { has a Weierstrass point of type } k\right\}
$$

and for $1 \leq k \leq g-2$ we shall set:

$$
E(k)=\left\{[C] \in M_{g} / C \text { has a Weierstrass point of type } g+k\right\},
$$

and

$$
F(k)=W(1,2, \ldots, g-1, g+k+1) .
$$

The locus $\mathbb{D}_{k}$ is non-empty, irreducible and of the "expected dimension" $2 g-3+k$ ([R], $[\mathrm{A}]$ ). The same as for $F(k)$, which is non-empty (for each $1 \leq k \leq g-2$ ) (see e.g. [Pi]) and has the expected dimension $3 g-3-k$ ([D2]). Moreover $F(k)$ is contained in $E(k)$ and $E(k)$ is hence non-empty. All the irreducible components of $E(k)$ have the same expected dimension $3 g-3-k([\mathrm{D} 2])$.

In the last two sections we shall deal with another locus in the moduli space, which for lack of a better notation will be denoted as $w t(k)(k \geq 1)$. It is defined as:

$$
w t(k)=\left\{[C] \in M_{g} / C \text { has a point } P \text { such that } w t(P) \geq k\right\} .
$$


1.5. Let $\pi: \mathfrak{X} \longrightarrow S$ be a smooth proper curve of genus $g$ over a smooth scheme $S$ of finite type over $\mathbb{C}$ (cf. section 4 for definitions). Let $K_{\pi}$ be the relative canonical sheaf (see section 2 for details). One can then define some classes in the Chow ring, $A^{*}(S)$, of $S$ :

$$
\kappa_{i}=\pi_{*}\left[c_{1}\left(K_{\pi}\right)^{i+1}\right],
$$

the so-called $\kappa$-classes and

$$
\lambda_{1}=\lambda=c_{1}\left(\pi_{*} K_{\pi}\right)=c_{1}\left(\bigwedge^{g} \pi_{*} K_{\pi}\right) \quad \text { and } \quad \lambda_{i}=c_{i}\left(\pi_{*} K_{\pi}\right),
$$

the so-called $\lambda$-classes.

As for definition (1.8), one has that (see [D1]) $\kappa_{0}=(2 g-2)[S]$, where $[S]$ is the fundamental class of $S$ (the identity of the ring $A^{*}(S)$ ). As for definitions (1.9), instead, we remark that $\pi_{*} K_{\pi}$ is a locally free rank $g$ sheaf of $O_{S}$ modules, often denoted in the literature as $\mathbb{E}$ and called the Hodge bundle relative to $\pi$.

Now let $M_{g, 1} \stackrel{\pi}{\longrightarrow} M_{g}$ be the natural morphism that "forgets" the marking. Notice that in this case, due to curves with non-trivial automorphisms, $M_{g}$ is no longer smooth for $g \geq 3$. However, via level $n$ structures, $M_{g}$ can be globally seen as the quotient of a smooth variety $\tilde{M}_{g}$ under a finite group $G$ acting faithfully on it (see, e.g., [OS], Theorem 1.8). This allows, following [Fu], p. 142, to define an intersection product on $A_{*}\left(M_{g}\right) \otimes \mathbb{Q}=\left[\bigoplus A_{i}\left(M_{g}\right)\right] \otimes \mathbb{Q}$ which makes $A_{*}\left(M_{g}\right) \otimes \mathbb{Q}$ itself into a ring. This will be, by definition, the Chow ring of $M_{g}$. In the following it will be denoted as $A^{*}\left(M_{g}\right)$, with contravariant notation. Moreover there is a proper flat family of curves $\tilde{\pi}: \tilde{\mathcal{C}}_{g} \longrightarrow \tilde{M}_{g}$, with $G$ acting faithfully on $\mathcal{C}_{g}$ compatibly with the action of $G$ on $\tilde{M}_{g}$ and the morphism $\pi$, such that $\tilde{\mathcal{C}}_{g} / G \cong M_{g, 1}$. Hence, following Mumford ([Mu2], p. 298), the morphism

$$
\pi: M_{g, 1} \longrightarrow M_{g}
$$

comes equipped with a $Q$-line bundle $K_{\pi}$, represented by the relative canonical bundle $K_{\tilde{\pi}}$, which shall be called briefly the relative canonical bundle of $\pi$, with no further mention. Accordingly, $\mathbb{E}=\pi_{*} K_{\pi}$ is a $Q$-bundle of rank $g$ over $M_{g}$, represented by $\tilde{\pi}_{*} K_{\tilde{\pi}}$ over $\tilde{M}_{g}$. The $\kappa$-classes and the $\lambda$ classes will be defined according to (1.8) and (1.9), where the Chern classes are taken in the sense of [Mu2]. The $\lambda$-classes and the $\kappa$-classes are said to be the tautological classes of $A^{*}\left(M_{g}\right)$ (cf. [Mu2]). It is worth mentioning that:

$$
A^{1}\left(M_{g}\right)=\operatorname{Pic}\left(M_{g}\right) \otimes \mathbb{Q} \cong \operatorname{Pic}\left(\mathcal{M}_{g}\right) \otimes \mathbb{Q},
$$

where the last right hand side means the Picard group (with $\mathbb{Q}$ coefficients) of the moduli functor $\mathcal{M}_{g}([\mathrm{HM}]$, p. 50).

1.6. In the following, and especially in section 3 and 4 below, when we speak of the class in $A^{*}\left(M_{g}\right)$ of a given subvariety of $M_{g}$, we shall always mean the $Q$-class of it. However, for short, we shall systematically skip the " $Q-"$, and we shall say classes with no further mention.

\section{Derivatives of Relative Wronskians}

2.1. The purpose of this section is to define a suitable notion of "derivative" of the relative wronskian in the sense, e.g., of [LT1], [LT2]. We recall briefly, for the reader's convenience, the basic framework which stays behind the extended notion of wronskians for families of curves. We limit ourselves to families of smooth curves, 
being enough for our purposes, although the same contruction can be performed dealing with families of stable curves, in the sense of [DM], and replacing the relative canonical sheaf by the relative dualizing sheaf (which is invertible in that case).

2.2. Our parameter spaces will be taken in the category of smooth schemes of finite type over the complex field $\mathbb{C}$. To start with, then, let $S$ be one such. By a smooth curve of genus $g \geq 2$ over $S$ we shall intend a flat proper surjective morphism $\pi: \mathfrak{X} \longrightarrow S$ such that the scheme theoretical fibers $\mathfrak{X}_{s}=\mathfrak{X} \times{ }_{S} \operatorname{Spec}(\mathbf{k}(s)):=\pi^{-1}(s)$ are smooth projective connected curves of genus $g$ over $\mathbf{k}(s) \cong \mathbb{C}$. In an analytic language, $\pi$ can be seen as a holomorphic proper map between complex analytic spaces, such that the fibers are compact Riemann surfaces of genus $g$. However, in this section we shall work mainly algebraically, since it is more convenient from a formal point of view.

2.3. As is well known, a smooth curve of genus $g$ over $S$ comes equipped with an invertible sheaf of $O_{\mathfrak{X}}$-modules, $\Omega_{\mathfrak{X} / S}^{1}([\mathrm{AK}], \mathrm{p}$. 108), the so-called sheaf of the relative differentials of $\mathfrak{X}$ over $S$, together with a universal derivation:

$$
d_{\mathfrak{X} / S}: O_{\mathfrak{X}} \longrightarrow \Omega_{\mathfrak{X} / S}^{1} .
$$

For notational convenience we shall denote, in the sequel, $\Omega_{\mathfrak{X} / S}^{1}$ by $K_{\pi}$, and $d_{\mathfrak{X} / S}$ by $d_{\pi}$. The bundle $K_{\pi}$ shall be called, in the following, the relative canonical sheaf with respect to $\pi$, and $d_{\pi}$ the exterior derivative along the fibers of $\pi$ (compare with [L1], where an analytic description of $d_{\pi}$ is provided). As is well known, $K_{\pi}$ and $d_{\pi}$ enjoy some nice functorial properties. More precisely, if:

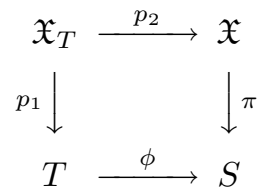

is a cartesian diagram, i.e. $\mathfrak{X}_{T}$ is the induced family over $T$, defined by $T \times_{S} \mathfrak{X}$, then $([\mathrm{AK}]$, p. 110):

$$
p_{2}^{*} K_{\pi}=K_{p_{1}}
$$

and

$$
p_{2}^{*} d_{\pi}=d_{p_{1}}
$$

In particular, if $s \in S$ and $T=\operatorname{Spec}(\mathbf{k}(s))$, then $p_{2}^{*} K_{\pi}=K_{p_{1}}=K_{\mathfrak{X}_{s}}$, the canonical bundle of the fiber $\mathfrak{X}_{s}$ and $d_{p_{2}} \equiv d: O_{\mathfrak{X}_{s}} \longrightarrow K_{\mathfrak{X}_{s}}$. This in particular means that $K_{\pi}$ is a canonical bundle along the fibers, i.e. its fiber at a point $P \in \mathfrak{X}$ is $K_{\mathfrak{X}_{\pi(P)}}$.

2.4. For pursuing our own goals we want to sketch the construction of what in [L1] is said to be the $n$-th jet bundle of $K_{\pi}$ along the fibers. There, the construction has been performed by using the Patt local coordinates ([Pa]) in the Teichmüller space. Following Lax in [L1], it will be denoted by $J_{\pi}^{n} K_{\pi}$.

So let $\mathcal{U}=\left\{U_{\alpha}: \alpha \in \mathcal{A}\right\}$ be an open affine covering of $\mathfrak{X}$ such that $K_{\pi}\left(U_{\alpha}\right)$ is generated by $\sigma_{\alpha}$ over $O_{\mathfrak{X}}\left(U_{\alpha}\right)$. Then if $\tau \in H^{0}\left(\mathfrak{X}, K_{\pi}\right)$, we can write $\tau_{\left.\right|_{\alpha}}=u_{\alpha} \sigma_{\alpha}$, for some $u_{\alpha} \in O_{\mathfrak{X}}\left(U_{\alpha}\right)$. The purpose now is to define the higher order derivatives with respect to the generator $\sigma_{\alpha}$ (compare, however, with [LT1] and [LT2]). We set $u_{\alpha}^{(0)}=u_{\alpha}$ and, recursively,

$$
d_{\pi}\left(u_{\alpha}^{(n-1)}\right)=u_{\alpha}^{(n)} \sigma_{\alpha}
$$


It is now a standard patching game to show that the collection:

$$
\left\{U_{\alpha} ; u_{\alpha}, u_{\alpha}^{\prime}, \ldots, u_{\alpha}^{(n)}\right\},
$$

defines a section, written $D^{n} \tau$, of a vector bundle which shall be denoted as $J_{\pi}^{n} K_{\pi}$ and which is exactly the $n$-th jet bundle of $K_{\pi}$ along the fibers of $\pi$ ([L1]), or the $(n+1)$-th sheaf of the principal parts relative to $\pi$ (see, e.g., [La]). Because of the property (2.4) of $d_{\pi}$, we also have, referring to the diagram (2.2),

$$
p_{2}^{*}\left(D^{k} \tau\right)=D^{k}\left(p_{2}^{*} \tau\right)
$$

and, hence:

$$
p_{2}^{*}\left(J_{\pi}^{k} K_{\pi}\right)=J_{p_{1}}^{k} K_{p_{1}}
$$

where the right hand side $D$ is exactly the $D$ relative to the induced family $p_{1}$ : $\mathfrak{X}_{T} \longrightarrow T$. In particular, if $T=\operatorname{Spec}(\mathbf{k}(s)), D$ is defined exactly as above and $J_{p_{1}}^{k} K_{p_{1}}$ the $k$-th jet of the canonical bundle of $\mathfrak{X}_{s}$.

2.5. As is explained in [LT1], on $\pi: \mathfrak{X} \longrightarrow S$ can be defined a wronskian, which is a section of the line bundle $K_{\pi}{ }^{\otimes \frac{g(g+1)}{2}} \otimes\left(\pi^{*} \bigwedge^{g} \pi_{*} K_{\pi}\right)^{\vee}$ over $\mathfrak{X}$ or, alternatively, a particular vector bundle morphism:

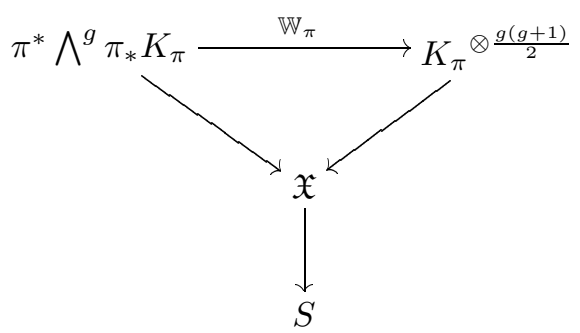

It is straightforward to show that such a relative wronskian restricts, fiberwise, to the usual wronskian. What we want to show, now, is that derivatives of arbitrary order of the above map can be taken. To do this, we shall perform a construction which includes, for $n=0$, as a particular case, the morphism (2.7) itself. More precisely we want to define morphisms:

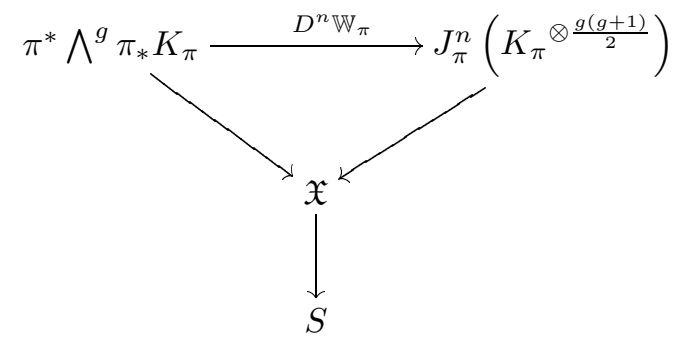

or, what is the same, sections $D^{n} \mathbb{W}_{\pi}$ of the vector bundle $J_{\pi}^{n}\left(K_{\pi} \otimes \frac{g(g+1)}{2}\right) \otimes$ $\left(\pi^{*} \bigwedge^{g} \pi_{*} K_{\pi}\right)^{\vee}$, that has rank $n+1$.

2.6. Construction. By well known theorems about the cohomology along the fibers of a morphism, it turns out that, in our situation, $\pi_{*} K_{\pi}$ is a locally free sheaf of $O_{S}$-modules of rank $g$ or, put otherwise, a rank $g$ vector bundle over $S$. Let $\mathcal{V}=\left\{V_{a}: a \in A\right\}$ be an affine covering of $S$ such that $\pi_{*} K_{\pi}$ is trivial on each $V_{a}$. Let $\boldsymbol{\omega}_{a}=\left(\omega_{1, a}, \omega_{2, a}, \ldots, \omega_{g, a}\right)$ be an $O_{S}\left(V_{a}\right)$-basis of $\pi_{*} K_{\pi}\left(V_{a}\right)$. Now, for each 
$i \in\{1, \ldots, g\}, \omega_{i, a} \in K_{\pi}\left(\pi^{-1}\left(V_{a}\right)\right)$. Since $\pi$ is proper, $\pi^{-1}\left(V_{a}\right)$ can be covered by a finite number of affine open sets $\mathcal{U}_{a}=\left\{U_{a \alpha}\right\}_{\alpha \in \mathcal{A}}$ (i.e. $\pi^{-1}\left(V_{a}\right)=\bigcup_{\alpha} U_{a \alpha}$ ) such that $K_{\pi}\left(U_{a \alpha}\right)$ is trivial over $O_{\mathfrak{X}}\left(U_{a \alpha}\right)$. Let $\sigma_{a \alpha}$ be a generator of $K_{\pi}\left(U_{a \alpha}\right)$ over $O_{\mathfrak{X}}\left(U_{a \alpha}\right)$ and define regular functions $u_{i, a \alpha} \in O_{\mathfrak{X}}\left(U_{a \alpha}\right)$ such that:

$$
\left.\omega_{i, a}\right|_{U_{a \alpha}}=u_{i, a \alpha} \sigma_{a \alpha}
$$

or, in a more concise form:

$$
\boldsymbol{\omega}_{\left.a\right|_{U_{a \alpha}}}=\mathbf{u}_{a \alpha} \sigma_{a \alpha},
$$

where $\mathbf{u}_{a \alpha}=\left(u_{1, a \alpha}, \ldots, u_{g, a \alpha}\right)$. Of course one can consider derivatives of the $u_{i, a \alpha}$ along the fibers in the sense of 2.4. Define:

$$
\mathbb{W}_{a \alpha}=\mathbf{u}_{a \alpha} \wedge \mathbf{u}_{a \alpha}^{\prime} \wedge \ldots \wedge \mathbf{u}_{a \alpha}^{(g-1)} .
$$

Recall that the notation of the right hand side simply means a determinant (cf. section 1), so that $\mathbb{W}_{a \alpha}$ is a regular function on $U_{a \alpha}$. As such it can be derived along the fibers, as explained in 2.4. Namely, we can use the relation:

$$
d_{\pi}\left(U_{a \alpha}\right)\left(\mathbb{W}_{a \alpha}\right)=\mathbb{W}_{a \alpha}^{\prime} \sigma_{a \alpha},
$$

as the intrinsic definition of the derivative $\mathbb{W}_{a \alpha}^{\prime}$. Similarly, $\mathbb{W}_{a \alpha}^{(n)}$ is recursively defined as:

$$
d_{\pi}\left(U_{a \alpha}\right)\left(\mathbb{W}_{a \alpha}^{(n-1)}\right)=\mathbb{W}_{a \alpha}^{(n)} \sigma_{a \alpha} .
$$

We consider now the set of all the data:

$$
\left\{D^{n} \mathbb{W}_{a \alpha}\right\}_{\alpha \in \mathcal{A}_{a}}=\left\{\left(U_{a \alpha} ; \mathbb{W}_{a \alpha}, \mathbb{W}_{a \alpha}^{\prime}, \ldots, \mathbb{W}_{a \alpha}^{(n)}\right): \alpha \in \mathcal{A}_{a}\right\}
$$

We have hence:

2.6. Theorem. The collection $\left\{D^{n} \mathbb{W}_{a \alpha}\right\}$ defines a global section, denoted as $D^{n} \mathbb{W}_{a}$, of a vector bundle of rank $n+1$ over $\pi^{-1}\left(V_{a}\right)$, which is precisely

$$
\left(J_{\pi}^{n} K_{\pi}^{\otimes \frac{g(g+1)}{2}}\right)_{\left.\right|_{\pi-1}\left(V_{a}\right)} .
$$

Proof. Consider the cartesian diagram:

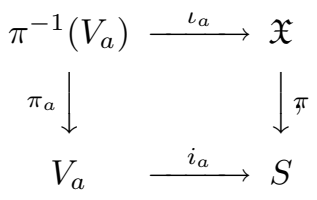

where $i_{a}$ and $\iota_{a}$ are the canonical immersion of $V_{a}$ and $\pi^{-1}\left(V_{a}\right)$ in $S$ and $\mathfrak{X}$ respectively. Set, as a notation, $K_{\pi_{a}}=\iota_{a}^{*}\left(K_{\pi}\right)$. By the functoriality of all the objects defined above and because the pull-back "commutes" with taking the tensor product, we have the following equalities:

$$
\begin{aligned}
& \iota_{a}^{*}\left(K_{\pi}^{\otimes N}\right)=\iota_{a}^{*}\left(K_{\pi}\right)^{\otimes N}=K_{\pi_{a}}^{\otimes N}, \\
& \iota_{a}^{*}\left(J_{\pi}^{h}\left(K_{\pi}^{\otimes N}\right)\right)=J_{\pi_{a}}^{h}\left(K_{\pi_{a}}^{\otimes N}\right),
\end{aligned}
$$

for all $N, h \geq 0$ (if $\mathcal{L}$ is any line bundle, we set $J^{0} \mathcal{L} \doteq \mathcal{L}$ ).

Suppose now that $U_{a \alpha} \cap U_{a \beta} \neq \emptyset$ and let, over such an intersection,

$$
\sigma_{\left.a \alpha\right|_{U_{a \alpha} \cap U_{a \beta}}}=\left.\chi_{\alpha \beta} \cdot \sigma_{a \beta}\right|_{U_{a \alpha} \cap U_{a \beta}} .
$$


The data $\left\{\chi_{\alpha \beta}\right\}_{(\alpha, \beta) \in \mathcal{A} \times \mathcal{A}}$ are nothing but the transition functions of $K_{\pi_{a}}$. Now, by direct computation, and using the skew-symmetry and the multilinearity of the determinant, one has:

$$
\mathbb{W}_{\left.a \alpha\right|_{U_{a \alpha} \cap U_{a \beta}}}=\left.\chi^{\frac{g(g+1)}{2}} \cdot \mathbb{W}_{a \beta}\right|_{U_{a \alpha} \cap U_{a \beta}} .
$$

This means that the $\mathbb{W}_{a \alpha}$ 's are the restrictions of a global holomorphic section $\mathbb{W}_{a}$ of $K_{\pi_{a}}^{\otimes \frac{g(g+1)}{2}}$, such that

$$
\mathbb{W}_{\left.a\right|_{U_{a \alpha}}}=\mathbb{W}_{a \alpha} \sigma_{a \alpha}
$$

Such a section is the wronskian section of the bundle $K_{\pi_{a}}^{\otimes \frac{g(g+1)}{2}}$.

Now, by 2.4 , if we consider, for each $n \geq 0$ the operators

$$
D^{n}: H^{0}\left(\pi^{-1}\left(V_{a}\right), K_{\pi_{a}}^{\otimes \frac{g(g+1)}{2}}\right) \longrightarrow H^{0}\left(\pi^{-1}\left(V_{a}\right), J_{\pi_{a}}^{n}\left(K_{\pi_{a}}^{\otimes \frac{g(g+1)}{2}}\right)\right),
$$

constructed by means of $d_{\pi_{a}}: O_{\pi^{-1}\left(V_{a}\right)} \longrightarrow K_{\pi_{a}}$, it turns out that, for each $n \geq 0$,

$$
D^{n} \mathbb{W}_{a} \in H^{0}\left(\pi^{-1}\left(V_{a}\right), J_{\pi_{a}}^{n}\left(K_{\pi_{a}}^{\otimes} \frac{g(g+1)}{2}\right)\right)
$$

2.7. End of the construction. After Theorem 2.6 the situation is now as follows. We are given a vector bundle $J_{\pi_{a}}^{n}\left(K_{\pi_{a}}^{\otimes} \frac{g(g+1)}{2}\right)$ on each open set $\pi^{-1}\left(V_{a}\right) \subseteq \mathfrak{X}$, together with a distinguished section of it, denoted by $D^{n} \mathbb{W}_{a}$. We now go back over the base $S$ of the family $\pi$, to observe that a holomorphic frame $\boldsymbol{\omega}_{a}$ of $\pi_{*} K_{\pi}\left(V_{a}\right)$ and a holomorphic frame $\boldsymbol{\omega}_{b}$ of $\pi_{*} K_{\pi}\left(V_{b}\right)$ are linked by the formula:

$$
\left(\boldsymbol{\omega}_{a}\right)=\left(G_{a b}\right)^{-1} \cdot\left(\boldsymbol{\omega}_{b}\right),
$$

where $\left\{G_{a b}\right\} \in Z^{1}\left(\mathcal{V}, G L_{g}\left(O_{S}\right)\right)$ are transition functions for the bundle $\pi_{*} K_{\pi}$. It follows that if $V_{a} \cap V_{b} \neq \emptyset$ and, hence, $\pi^{-1}\left(V_{a}\right) \cap \pi^{-1}\left(V_{b}\right) \neq \emptyset$, the following transformation rules hold:

$$
D^{n} \mathbb{W}_{a}=\pi^{*}\left(\operatorname{det}\left(G_{a b}^{-1}\right)\right) \cdot D^{n} \mathbb{W}_{b},
$$

where $\pi^{*}\left(\operatorname{det}\left(G_{a b}^{-1}\right)\right)$ means the regular function $\operatorname{det}\left(G_{a b}^{-1}\right)$ over $V_{a} \cap V_{b}$ extended to all $\pi^{-1}\left(V_{a} \cap V_{b}\right)$ in a fiberwise constant way. It is apparent that the $\pi^{*}\left(\operatorname{det}\left(G_{a b}^{-1}\right)\right)$ 's actually define a cocycle over the covering $\left\{\pi^{-1}\left(V_{a}\right): a \in A\right\}$, which is a set of transition functions for the line bundle $\left(\pi^{*} \bigwedge^{g} \pi_{*} K_{\pi}\right)^{\vee}$, the dual of $\pi^{*} \bigwedge^{g} \pi_{*} K_{\pi}$, over $\mathfrak{X}$. Hence all the data $\left\{\left(\pi^{-1}\left(V_{a}\right), D^{n} \mathbb{W}_{a}\right): a \in A\right\}$ define a global section, denoted as $D^{n} \mathbb{W}_{\pi}$, of $J_{\pi}^{n} K_{\pi} \otimes^{\frac{g(g+1)}{2}} \otimes\left(\pi^{*} \bigwedge^{g} \pi_{*} K_{\pi}\right)^{\vee}$, a vector bundle of rank $n+1$ over $\mathfrak{X}$. We have, thence:

2.8. Definition. The section $D^{n} \mathbb{W}_{\pi}$ of the bundle $J_{\pi}^{n} K_{\pi} \otimes^{\frac{g(g+1)}{2}} \otimes\left(\pi^{*} \bigwedge^{g} \pi_{*} K_{\pi}\right)^{\vee}$ constructed above, will be said to be the $n$-th derivative of the wronskian relative to the family $\pi: \mathfrak{X} \longrightarrow S . D^{0} \mathbb{W}_{\pi}$ is the usual wronskian as defined in [LT1] and [LT2]. The $n$-th derivative of the $\pi$-relative wronskian can be alternatively seen as 
a morphism of vector bundles:

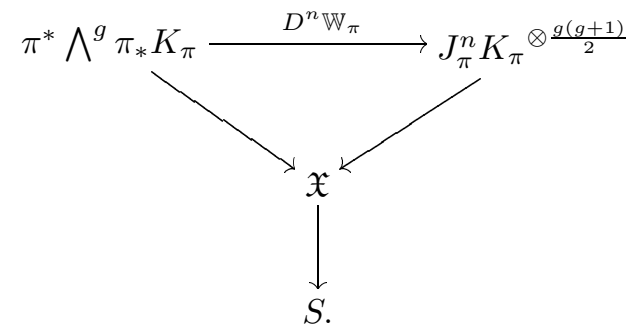

We need two more (easy) technical results. The former (Proposition 2.9) relates the relative wronskian, and its derivatives, with the Weierstrass points on the fibers of the family $\pi$ while the latter (Proposition 2.10) establishes a (well-known) exact sequence of vector bundles which shall be useful to compute Chern classes in sections 3 and 4 .

2.9. Proposition. Let $P$ be a point of $\mathfrak{X}$. Then $P$ is a Weierstrass point on $\pi^{-1}(\pi(P))$ of weight at least $k$, if and only if $D^{k-1} \mathbb{W}_{\pi}$ vanishes at $P$.

Proof. The key remark we use, here, is that the relative wronskian $\mathbb{W}_{\pi}$ restricted to a fiber $\mathfrak{X}_{s}$ is (up to a multiplicative constant) a wronskian section of the bundle $K_{s}^{\otimes \frac{g(g+1)}{2}}, K_{s}$ being the canonical sheaf of the fiber $\mathfrak{X}_{s}$. Let us denote by $\mathbb{W}_{s}$ such a restriction. Suppose now that $P$ is a WP for a fiber $\mathfrak{X}_{s}$ having weight at least $k$. Then

$$
D^{k-1} \mathbb{W}_{\pi}(P)=D^{k-1} \mathbb{W}_{s}(P),
$$

the second equality holding because of the functoriality of the wronskian. Now, the right hand side, in a local coordinate chart $(U, z)$ of the curve $\mathfrak{X}_{s}$, around the point $P$ and such that $z(P)=0$, can be expressed as the $k$-tuple:

$$
\left(w(0), w^{\prime}(0), \ldots, w^{(k-1)}(0)\right),
$$

where we set $\mathbb{W}_{\left.s\right|_{U}}=w(z) \cdot d z^{\otimes \frac{g(g+1)}{2}}$ and the derivatives are taken with respect to the local parameter $z$. By hypothesis $P$ is a WP of weight at least $k$; hence it is a zero of the wronskian of order $k$. Hence, the first $(k-1)$ derivatives of the wronskian, at $z=0$, must vanish, too. This proves the first implication.

Conversely, suppose that $D^{k-1} \mathbb{W}_{\pi}(P)=0$. Then, arguing as above, in a local coordinate chart around $P$ in the curve $\mathfrak{X}_{\pi(P)}$, the local expression of the wronskian vanishes at $P$ together with its $k-1$ derivatives, i.e. $P$ is a $\mathrm{WP}$ on $\mathfrak{X}_{\pi(P)}$ of weight at least $k$.

The next proposition is nothing but formula (2.1.1) in [LT1], p. 138, rephrased in the language of jets bundles. We shall refer to that paper for the proof, which is there established for a slightly more general case than the one that we presently need.

2.10. Proposition. For each line bundle $\mathcal{L}$ over $\mathfrak{X}$ and each $n \geq 1$, the following exact sequence holds:

$$
0 \longrightarrow \mathcal{L} \otimes K_{\pi}^{\otimes n} \longrightarrow J_{\pi}^{n} \mathcal{L} \stackrel{p_{n-1}}{\longrightarrow} J_{\pi}^{n-1} \mathcal{L} \longrightarrow 0
$$

In the next two sections we shall need such a proposition in the particular case in which $\mathcal{L}=K_{\pi}{ }^{\otimes \frac{g(g+1)}{2}}$. 


\section{Weierstrass POINTS OF WEIGHT AT LEAST $k$}

3.1. The purpose of this section is to begin the investigation of some very natural loci sitting inside the moduli space $M_{g}$, namely those characterized by curves having a WP of prescribed least weight. In other words we want to deal with the loci that, for each $k \geq 1$, are set-theoretically defined as in 1.4 :

$$
w t(k)=\left\{[C] \in M_{g} / C \text { possesses a Weierstrass point of weight at least } k\right\} .
$$

What we want to do, now, is to put on $w t(k)$ a suitable scheme structure when it is considered over the base of any smooth curve (cf. 2.2) of genus $g \geq 2$ over $S$ (a smooth scheme of finite type over $\mathbb{C}$ ). After the definitions set in section 2 , this turns out to be very natural, too. In fact, keeping the same notation of the last section, $w t(k)$ can be scheme-theoretically defined as:

$$
w t(k)=\pi\left(\mathcal{Z}\left(D^{k-1} \mathbb{W}_{\pi}\right)\right)
$$

where the symbol $\mathcal{Z}$ means that we are taking the zero scheme of the section

$$
D^{k-1} \mathbb{W}_{\pi} \in H^{0}\left(\mathfrak{X}, J_{\pi}^{k-1}\left(K_{\pi} \otimes \frac{g(g+1)}{2}\right) \otimes\left(\pi^{*} \bigwedge^{g} \pi_{*} K_{\pi}\right)^{\vee}\right) .
$$

The section $D^{k-1} \mathbb{W}_{\pi}$ can be locally written as a "vector" of $k$ local holomorphic functions, whose common zero locus cuts out precisely the points of $\mathfrak{X}$ having weight at least $k$ as WPs of the fiber where they do lie. Clearly, this is a closed set of $\mathfrak{X}$ and its projection onto $S$ gives rise to a closed set because $\pi$ is proper. Morally, we are fiberwise looking for special Weierstrass points having weight bigger than or equal to $k$, and patching them together to get the scheme $\mathcal{Z}\left(D^{k-1}\left(\mathbb{W}_{\pi}\right)\right)$. The following is one of the simplest but quite useful property of $w t(k)$.

3.2. Proposition. For each $1 \leq k \leq g-1$, the loci $E(k-1)$ and $\mathbb{D}_{g-k+1}$ are contained in $w t(k)$.

Proof. In fact, if $[C]$ belongs to $\mathbb{D}_{g-k+1}$, then $C$ has a WP whose first non-gap is smaller than or equal to $g-k+1$, and hence its weight is at least $k$. If $[C]$ belongs to $E(k-1)$, then it possesses a point $P$ of type $g+k-1$. Using the fact that (see $[\mathrm{Gu}]$ for references):

$$
h^{0}\left(C, O_{C}(d P)\right)=d+1-\sharp\{\text { Weierstrass gaps } \leq d\},
$$

it is easy to show that a point of type $g+k-1$ must have Weierstrass weight at least $k$, which completes the proof of the claim.

3.3. Of course, to each locus $\mathcal{Z}\left(D^{k-1}\left(\mathbb{W}_{\pi}\right)\right)$, we can associate a Chow class with $\mathbb{Q}$-coefficients in $A^{k-1}(S)$, by looking at $\pi_{*}\left[\mathcal{Z}\left(D^{k-1}\left(\mathbb{W}_{\pi}\right)\right)\right]$. Such a class will correspond to the actual class $[w t(k)]$ of the locus $w t(k)$ iff the codimension in $S$ of $w t(k)$ coincides with its expected codimension, i.e. to $k-1$. In this case $[w t(k)]$ can be computed by pushing down via $\pi$ the localized top Chern class ([Fu], p. 244) of the zero scheme associated to the section $D^{k-1}\left(\mathbb{W}_{\pi}\right)$. Simple examples show that it is too optimistic to hope that, in general, the expected codimension coincides with the actual one. For instance, the hyperelliptic locus clearly belongs to $w t(g(g-1) / 2)$, since it is cut out by $\pi\left(\mathcal{Z}\left(D^{\frac{g(g-1)}{2}}-1\left(\mathbb{W}_{\pi}\right)\right)\right)$. In this case, the expected codimension is $g(g-1) / 2-1$, while the actual codimension in $M_{g}$ is $g-2$. However, Proposition 3.2 may suggest that $w t(k)$ could have the expected dimension $3 g-2-k$ for all 
$1 \leq k \leq g-1$, i.e. in the same range where, by [R] and [D2], $E(k-1)$ and $\mathbb{D}_{g-k+1}$ do. But the situation can often be quite nasty, as it is shown below.

3.4. Counterexample. Let $k=4$ and $g=5$, and consider the locus $\mathbb{D}_{3} \subseteq M_{5}$. It is irreducible, non-empty and its dimension is 10 . The general element of $\mathbb{D}_{3}$ is a (isomorphism class of) curve having a WP with weight $\geq 4$. In fact, 3 is not a gap and then, by the semigroup property, 6 is a non-gap. The WGS at $P$ is $\left\langle 1,2,4,5, n_{5}\right\rangle$, with $7 \leq n_{5} \leq 8$, and hence $w t(P) \geq 4$. Hence $\mathbb{D}_{3}$ is an irreducible component of $w t(4)$, which has dimension 10, while the expected dimension of $w t(4)$ is 9. Similar examples can be easily constructed. For instance, $w t(5) \subset M_{7}$ has not the expected dimension, because it contains $\mathbb{D}_{4}$ as an irreducible component.

3.5. The localized top Chern class of $\mathcal{Z}\left(D^{k-1}\left(\mathbb{W}_{\pi}\right)\right)$ coincides with the actual class of the scheme $\mathcal{Z}\left(D^{k-1}\left(\mathbb{W}_{\pi}\right)\right)$ in $A^{k}(\mathfrak{X})$ if and only if such a zero scheme has the expected codimension $k$. Checking that is the same as checking that $w t(k)$ has the ex-

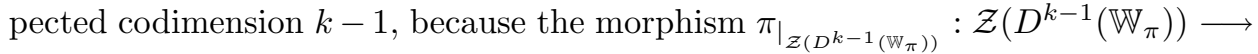
$w t(k)$ is finite. The previous counterexample shows, hence, that before pretending to compute the $\left(Q\right.$-)class of the locus $w t(k)$ in $A^{k-1}\left(M_{g}\right)$ by pushing down the localized top Chern class of $\mathcal{Z}\left(D^{k-1}\left(\mathbb{W}_{\pi}\right)\right)$, one must check case by case the equality of the expected dimension of $w t(k)$ with its actual dimension, if this is known for other geometrical reasons.

In this spirit, we think it would be interesting to find, for each $k \geq 2$, the least positive integer $g(k)$ such that $w t(k)$ has the expected dimension $3 g-k-2$ whenever $g \geq g(k)$. We have not yet a final answer to the above question but we offer, as contributions, Theorems 3.6 and 3.7 below.

3.6. Theorem. The locus wt(2) is empty in $M_{2}$. For $g>2$, each irreducible component of wt(2) has the expected dimension $3 g-4$.

Proof. For $g=2$ the claim is obvious, since no smooth curve of genus 2 has WP's with weight strictly bigger than 1 . For $g>2$ the proof relies on the fact that $[C] \in w t(2)$ if and only if either $[C] \in \mathbb{D}_{g-1}$ or $[C] \in E(1)$ (see the definitions in section 1). In fact, by Proposition 3.3., $E(1)$ and $\mathbb{D}_{g-1}$ are both contained in $w t(2)$. For checking the reversed inclusion, it suffices to show that if $P \in C$ has weight at least 2, then $P$ is a WP of type $g+1$ or has a first non-gap less than $g$. If the first non-gap at $P$ is $g-k(k \geq 1)$, then $[C] \in \mathbb{D}_{g-k} \subset \mathbb{D}_{g-1}$. If the first non-gap is equal to $g$, the point $P$ has weight at least 2 if it is a zero of the section $D \mathbb{W}_{\pi}$, i.e., by looking at the Brill-Nöther matrix, $\rho((g+1) P) \leq g-1$. Hence

$$
h^{0}\left(C, O_{C}((g+1) P)\right)=g+2-\rho((g+1) P) \geq 3,
$$

i.e. $P \in E(1)$. Now, by [D2], all the irreducible components of $E(1)$ have dimension $3 g-4$. Moreover, by $[\mathrm{R}]$ and $[\mathrm{A}], \mathbb{D}_{g-1}$ is irreducible and of dimension $3 g-4$ and the claim follows.

The above theorem was, more or less, already implicitly stated in the literature (cf. $[\mathrm{Cu}$, p. 339), so that the main result of this section (and, indeed, of this paper) consists in showing that $w t(3)$ has the same nice behaviour as $w t(2)$.

3.7. Theorem. Each irreducible component of wt(3) has the expected dimension $3 g-5$, for each $g \geq 4$. 
Proof. Let us start by checking what happens in genus 4 . In this case, the only WGSs having weight bigger than or equal to 3 , are $\langle 1,2,3,7\rangle,\langle 1,2,4,7\rangle$ and $\langle 1,3,5,7\rangle$. Each of them correspond to a WP of type $4+2=6$. This means that all the irreducible components of the loci $W(1,2,3,7), W(1,2,4,7), W(1,3,5,7)$ are contained in $E(2)$. But by [D2], all the irreducible components of $E(2)$ have codimension 2 in $M_{g}$, for $g \geq 4$.

For $g \geq 5$ we argue as follows. Let $n$ be the first non-gap of $\left\langle 1, n_{2}, \ldots, n_{g}\right\rangle$ and let $W\left(1, n_{2}, \ldots, n_{g}\right)$ be as in 1.4 , such that the weight of $\left\langle 1, n_{2}, \ldots, n_{g}\right\rangle$ as WGS is $\geq 3$. The set of all such $W\left(1, n_{2}, \ldots, n_{g}\right)$ 's can be divided into two disjoint subsets. On one hand we have the WGS for which $n \leq g-2$. Then the closures in $M_{g}$ of all such $W\left(1, n_{2}, \ldots, n_{g}\right), \overline{W\left(1, n_{2}, \ldots, n_{g}\right)}$, are contained in $\mathbb{D}_{g-2}$ which is irreducible and has the expected codimension 2. Or, we have the WGS's for which $n \geq g-2$, i.e. $n=g-1$ or $n=g(n=g+1$ gives rise to the normal WGS, while $n \geq g+2$ is impossible). Let us start by analyzing the case $n=g$. In this case, for having a WGS of weight $\geq 3$, the last gap $n_{g}$ must be strictly bigger than $g+2$. This means that $g+2$ is the third non-gap of such a sequence, and hence that $\overline{W\left(1,2, \ldots, g-1, n_{g} \geq g+3\right)}$ is contained in $E(2)$, whose irreducible components all have codimension 2. It remains to study what happens if $g-1$ is the first non-gap. The WGS's having $g-1$ as first non-gap and weight at least 3 are:

$$
\left\langle 1,2, \ldots, g-2, g, n_{g}\right\rangle
$$

with $n_{g} \geq g+2$ and

$$
\left\langle 1,2, \ldots, g-2, n_{g-1}, n_{g}\right\rangle
$$

with $n_{g-1} \geq g+1$ (i.e. $g$ is the second non-gap after the first non-gap $g-1$ ). As for the sequence $\left(^{*}\right)$, if $n_{g}=g+2$, then it has weight 3 . We claim that the codimension of $W(1,2, \ldots, g-2, g, g+2)$ is exactly 2 . In fact its codimension is at most 2, because the points of this set correspond to curves having WP's with weight 3 . Moreover it is contained in $\mathbb{D}_{g-1}$ which is irreducible and of codimension 1 . Now, $\overline{W(1,2, \ldots, g-2, g, g+1)}$ is non-empty, because its general element corresponds to a curve having a WP with weight $2 \leq[g / 2]$ (for $g \geq 5$ ), existing by $[\mathrm{EH}]$, p. 497. Such a locus has codimension at most 1 and hence exactly 1 . If $\overline{W(1,2, \ldots, g-2, g, g+2)}$ had codimension 1 , too, then $\mathbb{D}_{g-1}$ would not be irreducible. This proves that the codimension of $\overline{W(1,2, \ldots, g-2, g, g+2)}$ is exactly 2 .

If in the sequence $\left.{ }^{*}\right) n_{g} \geq g+3$, then $\overline{W\left(1,2, \ldots, g-2, g, n_{g}\right)}$ is contained in some irreducible component of $E(2)$.

It only remains to study the case $\left(^{* *}\right)$. But by a theorem of Rauch $([\mathrm{R}]$, Theorem 1, p. 545), the locus of curves in $M_{g}$ having $n$ and $n+1$ as first two non-gaps, if non-empty, has the expected dimension $2 g+n-4$, which for $n=g-1$ yields exactly $3 g-5$.

Summarizing the above results, we have checked that all the irreducible components of $w t(3)$ consist in $E(2), \mathbb{D}_{g-2}$ and in codimension 1 boundary components of $\mathbb{D}_{g-1}$.

This concludes the proof of the theorem. 
4. The LOCI $w t(2)$ And $w t(3)$ And SOME COMPUtATion IN $A^{i}\left(M_{g}\right)(i=1,2)$

4.1. The time has come, now, to show how the derivatives of the relative wronskians can be concretely used to perform computations in the Chow ring of $M_{g}$. The goal of this section will be to compute the class of $w t(2)$ in $A^{1}\left(M_{g}\right)$ (which, as recalled in section 1 , actually coincides with $\left.\operatorname{Pic}\left(M_{g}\right) \otimes \mathbb{Q}\right)$ and $w t(3)$ in $A^{2}\left(M_{g}\right)$. We shall discuss such results partly by revisiting some examples already worked out in the literature and partly to get some new hints on the geometry of $M_{g}$.

We first start with a simple example, which only involves the relative wronskian and no derivative of it. Such an example is the prototype of all the computations which shall be performed throughout this section.

4.2. Example (Cf. $[\mathrm{Cu}]$, p. 321 , or $[\mathrm{LT} 1]$ ). Let $\pi: \mathfrak{X} \longrightarrow S$ be a smooth proper curve of genus $g$ over $S$. Then the locus $\mathcal{W}_{\pi}$ of the Weierstrass points on the fibers of $\mathfrak{X}$ can be defined as $\mathcal{Z}\left(\mathbb{W}_{\pi}\right)$. By its very definition,

$$
\begin{aligned}
{\left[\mathcal{Z}\left(\mathbb{W}_{\pi}\right)\right] } & =c_{1}\left(K_{\pi}^{\otimes \frac{g(g+1)}{2}} \otimes\left(\pi^{*} \bigwedge^{g} \pi_{*} K_{\pi}\right)^{\vee}\right) \\
& =c_{1}\left(K_{\pi}^{\otimes \frac{g(g+1)}{2}}\right)-c_{1}\left(\pi^{*} \bigwedge^{g} \pi_{*} K_{\pi}\right) \\
& =\left(\begin{array}{c}
g+1 \\
2
\end{array}\right) c_{1}\left(K_{\pi}\right)-\pi^{*} \lambda,
\end{aligned}
$$

where $\lambda$ is the first Chern class of the Hodge bundle $\mathbb{E}=\pi_{*} K_{\pi}$.

4.3. From now on we begin to use the fact, proven in Theorem 3.5, that each irreducible component of $w t(2)$ has the expected dimension $3 g-4$. The scheme structure of $w t(2)$ is such that, by virtue of Proposition 2.9, the general element of an irreducible component having a WP of weight $k \geq 2$ must be counted with multiplicity at least $k-1$. To compute the class $[w t(2)]$ of $w t(2)$ in $\operatorname{Pic}\left(M_{g}\right) \otimes \mathbb{Q}$ it is sufficient to compute it on the base of a 1-parameter (proper, flat) family $\pi: \mathfrak{X} \longrightarrow S$ of smooth curves of genus $g$. This means that we are working on the moduli functor $\mathcal{M}_{g}([\mathrm{HM}]$, p. 50) and then we use Lemma 5.14 in [Mu1]. To perform such a computation it is sufficient to push down on $A^{1}(S)$, via $\pi$, the top Chern class of the rank 2 vector bundle $J_{\pi}^{1}\left(K_{\pi}^{\otimes \frac{g(g+1)}{2}} \otimes\left(\pi^{*} \bigwedge^{g} \pi_{*} K_{\pi}\right)^{\vee}\right)$. We have, hence, using standard properties of Chern classes:

$$
\begin{aligned}
\pi_{*}\left[\mathcal{Z}\left(D \mathbb{W}_{\pi}\right)\right] & =\pi_{*} c_{2}\left[J_{\pi}^{1} K_{\pi} \otimes \frac{g(g+1)}{2} \otimes\left(\left(\pi^{*} \bigwedge^{g} \pi_{*} K_{\pi}\right)^{\vee}\right)\right] \\
& =\pi_{*} c_{2}\left(J_{\pi}^{1} K_{\pi} \otimes \frac{g(g+1)}{2}-\pi^{*} \bigwedge^{g} \pi_{*} K_{\pi}\right),
\end{aligned}
$$

where the "difference" is taken in the Grothendieck group of relative coherent sheaves on $\mathfrak{X} / S$. One has:

$$
\begin{aligned}
& \pi_{*} c_{2}\left(J_{\pi}^{1} K_{\pi}^{\otimes \frac{g(g+1)}{2}}-\pi^{*} \bigwedge_{*}^{g} \pi_{\pi} K_{\pi}\right. \\
& =\pi_{*}\left[c_{2}\left(J_{\pi}^{1} K_{\pi} \otimes \frac{g(g+1)}{2}\right)-c_{1}\left(J_{\pi}^{1} K_{\pi} \otimes \frac{g(g+1)}{2}\right)\right] \pi^{*} \lambda \\
& =\pi_{*}\left\{\left[\frac{g(g+1)}{2}+1\right] \frac{g(g+1)}{2} c_{1}\left(K_{\pi}\right)^{2}-[g(g+1)+1] \pi^{*} \lambda c_{1}\left(K_{\pi}\right)\right\},
\end{aligned}
$$


where the last equality is gotten by applying the exact sequence (2.15) to the bundle $K_{\pi}^{\otimes \frac{g(g+1)}{2}}$ for $n=2$ and $n=1$, and taking the Chern classes. Now, by using the push-pull formula and simplifying all the expressions involved:

$$
[w t(2)]=\frac{1}{4} g\left(g^{3}+2 g^{2}+3 g+2\right) \kappa_{1}-2\left(g^{3}-1\right) \lambda .
$$

The above relation holds in the Picard group $\operatorname{Pic}\left(\mathcal{M}_{g}\right)$ of the moduli functor of smooth curves $\mathcal{M}_{g}\left([\mathrm{HM}]\right.$, p. 50), i.e. by $[\mathrm{Mu} 1]$, in $\operatorname{Pic}\left(M_{g}\right) \otimes \mathbb{Q}$. For $g=2$ the above expression gives the relation:

$$
0=\pi_{*} \mathcal{Z}\left(D \mathbb{W}_{\pi}\right)=12 \kappa_{1}-14 \lambda
$$

(i.e., there are no WP's of weight 2 or more on a curve of genus 2), which together with the expression $\kappa_{1}=2 \lambda\left([\mathrm{Mu} 2]\right.$, p. 316) yields $\kappa_{1}=\lambda=0$ in $\operatorname{Pic}\left(M_{2}\right) \otimes \mathbb{Q}$. This is in agreement with the fact that $\lambda$ is supported, on $\bar{M}_{2}$, only on the boundary, as was well known.

4.4. For $g=3$, formula (4.2) gives:

$$
[w t(2)]=42 \kappa_{1}-52 \lambda
$$

holding in $\operatorname{Pic}\left(M_{3}\right) \otimes \mathbb{Q}$. Using the relation $\kappa_{1}=12 \lambda([\mathrm{Mu} 1]$, p. 102, or $[\mathrm{D} 1]$, p. 7$)$, one gets $[w t(2)]=452 \lambda$. We already know that $w t(2)$ is a divisor in $M_{g}$. In genus 3 it is exactly the set theoretical union of the closure of the hyperelliptic locus and of the closure of curves having at least 1 hyperflex (i.e. a WP with WGS $=\langle 1,2,5\rangle$ ). The scheme structures of the latter subsets has been defined in [Mu2], p. 310, and [D1], p. 59, respectively. Hence, in $\operatorname{Pic}\left(M_{3}\right) \otimes \mathbb{Q}$ we must have the relation:

$$
[w t(2)]=a[W(1,2,5)]+8 b\left[H_{3}\right]
$$

for some integers $a, b \geq 1$. The factor 8 is due to the fact that $\pi^{-1}\left(H_{3}\right)$ covers $H_{3}$ $8: 1$, while $b$ must be at least 2 . This is because a hyperelliptic point $P$ has Weierstrass weight 3 , and hence $(C, P) \in M_{3,1}$, with $P$ a Weierstrass point on the hyperelliptic curve $C$, is a zero of order at least 2 of the "derivative" of the wronskian $D \mathbb{W}_{\pi}$, as follows from Proposition 2.9. Actually, by a purely computational argument we may conclude that $a=1$ and $b=2$. In fact the $\left(Q\right.$-)class of $H_{3}$ (the hyperelliptic locus) in $M_{3}$ is known to be $9 \lambda$ while by [Cu], pp. 343-344, or basically [D1], p. 59 , the class $[V]$ of the hyperflex divisor in $M_{3}$ is given by:

$$
[V]=308 \lambda \text {. }
$$

The equality:

$$
[w t(2)]=[W(1,2,5)]+16\left[H_{3}\right],
$$

comes now from the fact that $452=308+16 \cdot 9$.

4.5. For $g>3, w t(2)$ is the set theoretical union of the closure of curves having a WP with gap sequence $\langle 1,2, \ldots, g-1, g+2\rangle$ and a WP with gap sequence 
$\langle 1,2, \ldots, g-2, g, g+1\rangle$, i.e. the only two gaps sequences having weight 2 . The $Q$-class of each of them occurs with multiplicity at least 1 (they belong to the zero scheme of $D \mathbb{W}_{\pi}$ !) in the class of $[w t(2)]$, and they actually correspond to the classes $\left[\mathbb{D}_{g-1}\right]$ and $[E(1)]$ respectively. Now we know, by [D1], that:

$$
\left[\mathbb{D}_{g-1}\right]=\frac{g^{2}(g-1)(3 g-1)}{2} \lambda,
$$

and that:

$$
[E(1)]=\frac{(g+1)(g+2)\left(3 g^{2}+3 g+2\right)}{2} \lambda .
$$

It is now a trivial matter to check that:

$$
\left(3 g^{4}+4 g^{3}+9 g^{2}+6 g+2\right) \lambda=[w t(2)]=[E(1)]+\left[\mathbb{D}_{g-1}\right] .
$$

We have hence proven that:

4.6. Theorem. For $g \geq 4$, the following equality holds in the group $\operatorname{Pic}\left(M_{g}\right) \otimes \mathbb{Q}$ :

$$
[w t(2)]=[E(1)]+\left[\mathbb{D}_{g-1}\right],
$$

while, for $g=3$, one has:

$$
[w t(2)]=[W(1,2,5)]+16\left[H_{3}\right]
$$

in $\operatorname{Pic}\left(M_{3}\right) \otimes \mathbb{Q}$.

4.7. Remarks. a) The equalities (4.7) and (4.8) are meaningful thanks to the fact that we have been able to equip the set $w t(2)$ with a scheme structure. Moreover, Theorem 4.6 shows that such a scheme structure is very natural, because it gives back equalities holding already in the set theoretic framework. As we shall see below, things become more difficult, already in genus 4, for the scheme $w t(3)$.

b) Let $\overline{M_{g}}(g \geq 3)$ be the Deligne-Mumford compactification of $M_{g}$ and let $\overline{w t(2)}$ be the closure of $w t(2)$ in $\overline{M_{g}}$. It is very easy, as shown in [Ga3], to compute the class, $[\overline{w t(2)}]$, in $\operatorname{Pic}\left(\bar{M}_{g}\right) \otimes \mathbb{Q}$ of $\overline{w t(2)}$. The only thing to do is to learn how to use the derivative of the relative wronskian, in the sense of Section 2, for families of curves with singular (stable) fibers, using the relative version of the jets bundles over (Gorenstein) singular curves treated in [Ga1] and [Ga2].

Analogously, let $\overline{\mathbb{D}_{g-1}}$ and $\overline{E(1)}$ be the closure in $\bar{M}_{g}$ of $\mathbb{D}_{g-1}$ and $E(1)$ respectively. By the exact sequence of additive groups ([Mu2], p. 307 or, in general, [Fu], p. 21):

$$
A^{*}\left(\overline{M_{g}} \backslash M_{g}\right) \longrightarrow A^{*}\left(\overline{M_{g}}\right) \longrightarrow A^{*}\left(M_{g}\right) \longrightarrow 0,
$$

it follows that, for $g \geq 4,[\overline{w t(2)}]$ is equal, by Theorem 4.6 , to the sum $[\overline{E(1)}]+\left[\overline{\mathbb{D}_{g-1}}\right]$ modulo boundary components. In [Ga3] it is shown that actually, the following equality holds:

$$
[\overline{w t(2)}]=[\overline{E(1)}]+\left[\overline{\mathbb{D}_{g-1}}\right]
$$

and using the main theorem of $[\mathrm{D} 1]$, expressing the class $\left[\overline{\mathbb{D}_{g-1}}\right]$ in $\operatorname{Pic}\left(\overline{M_{g}}\right) \otimes \mathbb{Q}$ and the easy computability of $\overline{w t(2)}$ (due to the use of a "derivative of a wronskian"), this provides, as a by-product, an easier proof of the main theorem of $[\mathrm{Cu}]$, 
expressing the class of $\overline{E(1)}$. For $g=3$ one has instead:

$$
[\overline{w t(2)}]=\overline{W(1,2,5)]}+16\left[\overline{H_{3}}\right]
$$

(See [Ga3] for details.)

4.8. The fact that, as proven in Theorem 3.7, all of the irreducible components of the locus $w t(3) \subset M_{g}$ have, for $g \geq 4$, the same expected dimension $3 g-5$, has as a first consequence the computability of the $Q$-class of $[w t(3)]$ in $A^{2}\left(M_{g}\right)$. As usual, by the very definition of $Q$-class, the computation can be performed putting ourselves in the following situation: $\pi: \mathfrak{X} \longrightarrow S$ is a smooth curve of genus $g \geq 4$ over a smooth surface $S$ (in the sense of 2.2 ), and $D^{2} \mathbb{W}_{\pi}$ is the second derivative of the relative wronskian $\mathbb{W}_{\pi}$. The calculations work exactly as in the previous subsections.

4.9. Proposition. If $\lambda, \kappa_{1}$ and $\kappa_{2}$ are the classes computed out of the $Q$-line bundle $K_{\pi}$ on $M_{g, 1}$, the $Q$-class of wt(3) is given by:

$$
\begin{aligned}
{[w t(3)] } & =\frac{1}{8} g(g+1)\left(g^{2}+g+2\right)\left(g^{2}+g+4\right) \kappa_{2}+ \\
& -\frac{1}{4}\left[2 g(g+1)\left(g^{2}+g+3\right)+\left(g^{2}+g+2\right)\left(g^{2}+g+4\right)\right] \kappa_{1} \lambda .
\end{aligned}
$$

Proof. In fact, $w t(3)=\pi\left(\mathcal{Z}\left(D^{2} \mathbb{W}_{\pi}\right)\right)$, and $D^{2} \mathbb{W}_{\pi}$ is a section of $J_{\pi}^{2} K_{\pi} \otimes \frac{g(g+1)}{2} \otimes$ $\left.\left(\pi^{*} \bigwedge^{g} \pi_{*} K_{\pi}\right)^{\vee}\right)$. Hence:

$$
\begin{aligned}
{[w t(3)] } & =\pi_{*}\left[c_{3}\left(J_{\pi}^{2} K_{\pi}^{\otimes \frac{g(g+1)}{2}} \otimes\left(\pi^{*} \bigwedge^{g} \pi_{*} K_{\pi}\right)^{\vee}\right)\right] \\
& =\pi_{*} c_{3}\left(J_{\pi}^{2} K_{\pi}{ }^{\frac{g(g+1)}{2}}-\pi^{*} \bigwedge^{g} \pi_{*} K_{\pi}\right) \\
& =c_{3}\left(J_{\pi}^{2} K_{\pi} \otimes \frac{g(g+1)}{2}\right)-c_{2}\left(J_{\pi}^{2} K_{\pi} \otimes \frac{g(g+1)}{2}\right) \pi^{*} \lambda,
\end{aligned}
$$

where, as usual, the "difference" occurring in the second equality is taken in the Grothendieck group of the coherent sheaves of $\mathfrak{X} / S$. Now we use the following two exact sequences, inferred from Proposition 2.9:

$$
0 \longrightarrow K_{\pi}^{\otimes\left(\frac{g(g+1)}{2}+2\right)} \longrightarrow J_{\pi}^{2} K_{\pi}^{\otimes}{ }^{\frac{g(g+1)}{2}} \longrightarrow J_{\pi}^{1} K_{\pi} \otimes^{\frac{g(g+1)}{2}} \longrightarrow 0,
$$

and

$$
0 \longrightarrow K_{\pi}^{\otimes\left(\frac{g(g+1)}{2}+1\right)} \longrightarrow J_{\pi}^{1} K_{\pi}^{\otimes \frac{g(g+1)}{2}} \longrightarrow K_{\pi}^{\otimes \frac{g(g+1)}{2}} \longrightarrow 0,
$$

in order to compute the second and the third Chern class of $J_{\pi}^{2} K_{\pi} \otimes \frac{g(g+1)}{2}$ occurring in the last side of (4.9). One gets easily, after some quick computation:

$$
\begin{aligned}
{[w t(3)] } & =\pi_{*}\left[\frac{1}{8} g(g+1)\left(g^{2}+g+2\right)\left(g^{2}+g+4\right) c_{1}\left(K_{\pi}\right)^{3}+\right. \\
& \left.-\frac{1}{4}\left[2 g(g+1)\left(g^{2}+g+3\right)+\left(g^{2}+g+2\right)\left(g^{2}+g+4\right)\right] c_{1}\left(K_{\pi}\right)^{2} \pi^{*} \lambda\right],
\end{aligned}
$$

i.e., after pushing down each summand, exactly formula (4.9). 
4.10. Proposition 4.9 above allows us to make a few remarks on the geometry of some loci of $M_{4}$. For instance, by their very definitions, it turns out that, as a set, $w t(3)$ coincides with $E(2)$ in genus 4 . This is because the only $W G S$ having weight at least 3 are $\langle 1,3,5,7\rangle$ (corresponding to Weierstrass points of hyperelliptic curves), $\langle 1,2,4,7\rangle$ and $\langle 1,2,3,7\rangle$. Moreover, all the points having one of the mentioned gap sequences are points of type $g+2$ (see section 1 for definitions). Conversely, if $[C] \in M_{4}$ falls in $E(2)$, then it must fall in one of the sets $W(1,2,3,7), W(1,2,4,7)$ or in $H_{4}$, the hyperelliptic locus. Now, basically by [D2], the fundamental class of the locus $E(2)$ may be easily computed: here the scheme structure of the WP's of type $6=4+2$ on fibers of $\mathfrak{X}$ is locally defined by the ideal $\mathcal{I}$ generated by the minors of the matrix which locally represents the natural evaluation map:

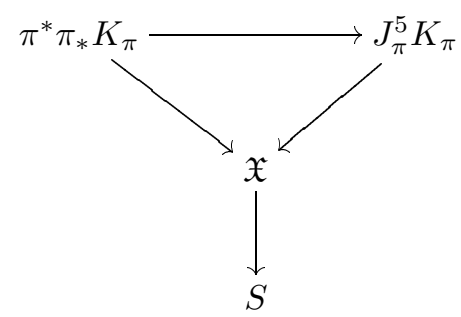

when its rank is smaller than or equal to 3. Applying Porteous's formula one gets $(g \geq 4)$ :

$$
[E(2)]=735 \kappa_{2}-175 \kappa_{1} \lambda_{1}+126 \lambda_{2} .
$$

Recall that in ([Fa1]) it is proven that the tautological ring of $M_{4}$ is generated by $\kappa_{1}$. In $A^{*}\left(M_{4}\right)$ the following relation holds ([Fa1]):

$$
\kappa_{1}^{2}=\frac{32}{3} \kappa_{2} .
$$

Moreover Mumford, in [Mu1], [Mu2], proves that:

$$
\lambda=\frac{\kappa_{1}}{12} \quad \text { and } \quad \lambda_{2}=\frac{\kappa_{1}^{2}}{288} .
$$

By substituting such equalities in formula (4.9) and formula (4.12) one sees that:

$$
[w t(3)] \neq[E(2)]
$$

so that the respective scheme structures of $w t(3)$ and $E(2)$ are certainly different. Of course, one may try to show this fact by directly comparing the ideal sheaves defining such schemes, but this turns out to be combinatorially quite tricky and it requires the knowledge of some properties of the wronskians which will appear elsewhere.

4.11. Concluding remarks. One of our original hopes was to be able to compute the classes in $A^{2}\left(M_{g}\right)$ of the two loci $W(1,2,4,7)$ and $W(1,2,3,7)$, by imitating the underlying philosophy of Theorem 4.6 and Remark 4.7. In fact, for suitable positive integers, $a, b, c, d, e, f$, the following equalities hold in the group $A^{2}\left(M_{g}\right)$ :

$$
[w t(3)]=10 \cdot a\left[H_{4}\right]+b[W(1,2,4,7)]+c[W(1,2,3,7)],
$$

and

$$
[E(2)]=10 \cdot d\left[H_{4}\right]+e[W(1,2,4,7)]+f[W(1,2,3,7)]
$$


Once one knows the exact value of the coefficients occurring in the above expressions, the classes $[w t(3)],[E(2)]$ and $\left[H_{4}\right]$ being known, the classes $[W(1,2,4,7)]$ and $[W(1,2,3,7)]$ would be gotten by simply solving a linear system of equations. Even if we do not know the exact value of the above coefficients, we may give some estimates on the $a$ and $b$. We know, in fact, that $a \geq 4$ and $b \geq 2$. This is because on a smooth proper curve over $S, \pi: \mathfrak{X} \longrightarrow S$, and $S$ has dimension at least 2, the locus of WP's on fibers of $\mathfrak{X}$ having weight at least 3 occurs as zeros of the section $D^{2}\left(\mathbb{W}_{\pi}\right)$, whose restriction to a fiber over $s \in S$ is exactly $D^{2}\left(\mathbb{W}_{\pi^{-1}(s)}\right)$. This means that if the Weierstrass weight of $P_{s} \in \pi^{-1}(s)$ is $2+h(h \geq 0)$, then it occurs in $\mathcal{Z}\left(D^{2}\left(\mathbb{W}_{\pi}\right)\right)$ with multiplicity at least $h$. Hence in $\mathcal{Z}\left(D^{2}\left(\mathbb{W}_{\pi}\right)\right)$, the locus of points on fibers of $\mathfrak{X}$ with gaps sequence $\langle 1,2,3,7\rangle$ occurs with multiplicity at least 1 , those with WGS $\langle 1,2,4,7\rangle$ occurs with multiplicity at least 2 and, finally, the hyperelliptic ones (WGS $=\langle 1,3,5,7\rangle)$ with multiplicity at least 4 . As should be clear we have basically used Proposition 2.9. Now by [D2] and [Co1] the locus of points $P \in \mathfrak{X}$ having $\langle 1,2,3,7\rangle$ or $\langle 1,2,4,7\rangle$ as a WGS projects onto $S$ generically 1-1 (i.e. the general curve having a WP of the type considered have all the other WP's normal). On the other hand the locus of hyperelliptic points of fibers of $\mathfrak{X}$ projects $10: 1$ onto the class of $\left[H_{4}\right]$ (the general fiber over $[C] \in H_{4}$ has 10 hyperelliptic WP's). Pushing down those loci to $S$, we have then explained (4.13). A similar reasoning yields the expression (4.14) for $E(2)$.

We should say that we hoped, indeed, to be able to compute exactly the coefficients $a, b, c, d, e, f$. We actually claimed, in a preprint form of this paper, that $a=4, b=2$ and $c=d=e=f=1$, but our proof contained a mistake, as pointed out to us by Marc Coppens ([Co2]).

Hence the problem of the computation of the coefficients occurring in equalities (4.13) and (4.14) remains open. After some first attempts to compute them, we realized that such computations are certainly interesting from a theoretical point of view, because they involve a fine knowledge of the local expressions of the relative wronskians and, we may now say, of its derivatives, which of course reflects the geometry of the Weierstrass scheme itself. Such a local structure is trivial for families parametrized by a point (a single curve!) and is relatively easy, and basically known in the literature, for families parametrized by a curve. As the above example shows, things become more serious and less clear for 2-parameters families of smooth curves, and the investigation of the local expression of the relative wronskian and its derivatives will be the subject of further researches.

\section{REFERENCES}

[A] E. Arbarello, Weierstraß Points and Moduli of Curves, Compositio Mathematica 29 (1974), 325-342. MR 50:13048

[ACGH] E. Arbarello, M. Cornalba, P.A. Griffiths, J. Harris, Geometry of Algebraic Curves, Vol 1, Springer-Verlag, 1985. MR 86h:14019

[AK] A. Altman, S. Kleiman, Introduction to Grothendieck Duality Theory, Lectures Notes in Mathematics, 146, Springer-Verlag, 1970. MR 43:224

[Co1] M. Coppens, The number of Weierstrass points on some special curve I, Arch. Math. 46 (1986), 453-465. MR 87h:14022

[Co2] M. Coppens, Private communication, (1997).

$[\mathrm{Cu}]$ F. M. Cukierman, Families of Weierstrass Points, Duke Math. J. 58, no. 2 (1989), 317-346. MR 90g:14013

[D1] S. Diaz, Exceptional Weierstrass points and the divisor on moduli space that they define, Vol 56, number 327, Memoirs of the American Mathematical Society, 1985. MR 86j: 14022 
[D2] S. Diaz, Tangent spaces in moduli via deformations with applications to Weierstrass points, Duke Math. J. 51 (1984), 905-922. MR 86d:14023

[DM] P. Deligne, D. Mumford, The irreducibility of the space of curves of given genus, Publ. Math. I.H.E.S. 36 (1969), 75-109. MR 41:6850

[EH] D. Eisenbud, J. Harris, Existence, decomposition and limits of certain Weierstrass points, Invent. Math. 87 (1987), 495-515. MR 88a:14028b

[Fa1] C. Faber, Chow rings of moduli spaces of curves II: Some results on the Chow ring of $\overline{\mathcal{M}}_{4}$, Ann. of Math. 132 (1990), 421-449. MR 91h:14009b

[Fa2] C. Faber, A Conjectural Description of the Moduli Space of Curves, "AmsterdamUtrecht seminar on moduli spaces of curves" (C. Faber, E.Looijenga, eds.), A. \& K. Peters Inc., to appear.

[Fo] O. Forster, Lectures on Riemann Surfaces, Springer-Verlag, 1984.

[Fu] W. Fulton, Intersection Theory, Springer-Verlag, 1984. MR 85k:14004

[Ga1] L. Gatto, Weight Sequences versus Gap Sequences at Singular Points of Gorenstein Curves, Geometriae Dedicata 54 (1995), 267-300. MR 96e:14031

[Ga2] L. Gatto, Weierstrass Loci and Generalizations, I, in "Projective Geometry with Applications" (E. Ballico, ed.), Marcel Dekker, Inc., 1994, pp. 137-166. MR 95m:14024

[Ga3] L. Gatto, On the closure in $\bar{M}_{g}$ of smooth curves having a special Weierstrass point, Math. Scand., to appear.

[GP] L. Gatto, F. Ponza, Generalized Wronskian Sections and Families of Weierstrass Points with Prescribed Minimal Weight, Rapporto Interno, Politecnico di Torino, n. 31 (1996), (hard copies available upon request).

[Gu] R.C. Gunning, Lectures on Riemann Surfaces, Princeton Mathematical Notes, 1966. MR 34:7789

[Ha] Robin Hartshorne, Algebraic Geometry, Springer-Verlag, 1977. MR 57:3116

[HM] J. Harris, D. Mumford, On the Kodaira Dimension of the Moduli Space of Curves, Invent. Math. 67 (1982), 23-88. MR 83i:14018

[La] D. Laksov, Weierstrass points on curves, Astérisque 87-88 (1981), 221-247. MR 83e: 14023

[L1] R.F. Lax, Weierstrass Points of the Universal Curve, Math. Ann. 216 (1975), 35-42. MR 52:5681

[L2] R.F. Lax, Gap Sequences and Moduli in Genus 4, Math. Z. 175 (1980), 67-75. MR 82a:32030

[LT1] D. Laksov, A. Thorup, The Brill-Segre Formula for Families of Curves, in "Enumerative Algebraic Geometry:Proceedings of the 1989 Zeuthen Symposium" (S.L. Kleiman and A. Thorup, eds.), Contemporary Mathematics, vol. 123, AMS, Providence, 1991, pp. 131-148. MR 92k:14029

[LT2] D. Laksov, A. Thorup, Weierstrass points and gap sequences for families of curves, Ark. Mat. 32 (1994), 393-422. MR 96b:14041

[Mu1] D. Mumford, Stability of Projective Varieties, L'Ens. Math. 23 (1977), 39-110. MR 56: 8568

[Mu2] D. Mumford, Towards an Enumerative Geometry of the Moduli Space of Curves, Progress in Math. 36 (1983), 271-328. MR 85j:14046

[OS] F. Oort, J. Steenbrink, The local Torelli problem for algebraic curves, Geom. Alg. Angers 1979, 1980, pp. 157-204. MR 82i:14014

[Pa] Charles Patt, Variations of Teichmueller and Torelli Surfaces, J. Anal. Math. 11 (1963), 221-247. MR 28:4103

[Pi] Deformation of Algebraic Varieties with $G_{m}$-action, (Astérisque 2a).

[Po1] F. Ponza, Sezioni wronskiane generalizzate e famiglie di punti di Weierstrass, Doctoral Thesis, Consorzio Universitario Torino-Genova, 1997.

[Po2] F. Ponza, Generalized Wronskian Sections and their Zero-Loci, in preparation (1997).

[R] H.E. Rauch, Weierstraß Points, Branch Points, and Moduli of Riemann Surfaces, Comm. in Pure and Applied Mathematics XII (1959), 543-560. MR 22:1666

Dipartimento di Matematica, Politecnico di Torino, Corso Duca degli Abruzzi, 2410129 TORINO, ITALY

E-mail address: lgatto@polito.it

E-mail address: ponza@dm.unito.it 